\title{
THE SZEGÖ PROJECTION: SOBOLEV ESTIMATES IN REGULAR DOMAINS
}

\author{
HAROLD P. BOAS
}

\begin{abstract}
The Szegö projection preserves global smoothness in weakly pseudoconvex domains that are regular in the sense of Diederich, Fornæss, and Catlin. It preserves local smoothness near boundary points of finite type.
\end{abstract}

The Bergman projection, being linked to the $\bar{\partial}$ problem, plays an important role in several complex variables, as demonstrated by recent applications to the boundary behavior of holomorphic maps $[7,16]$. The Szegö projection is interesting both by analogy and by its connection with the $\bar{\partial}_{b}$ problem and hence with unsolvable equations of Lewy type. While in strictly pseudoconvex domains the Bergman and Szegö projections are both well understood, in general much more is known about the Bergman projection. This is no surprise, for the theory of the $\bar{\partial}$ problem is further advanced than the theory of the $\bar{\partial}_{b}$ problem. Nonetheless I believe that the Szegö projection ought not be any more mysterious than the Bergman projection. This paper is intended to support this contention.

The most important domains for which the Bergman projection is known to be regular are domains with a good $\bar{\partial}$-Neumann operator. Here it is shown that in such domains the Szegö projection also is regular. More precisely, in smooth bounded pseudoconvex domains satisfying Catlin's property (P) (the most general condition presently known that guarantees global regularity of the Neumann operator) the Szegö projection is shown to be globally regular (Theorem 4.1). Moreover at boundary points of finite type the Szegö projection is locally regular (Theorem 5.1). In both cases the Szegö projection exactly preserves the degree of differentiability, measured in Sobolev norms.

The method of proof is not the obvious one of directly generalizing from the strictly peudoconvex case, for there are serious obstacles to such a generalization. For instance the approach of analysis on the Heisenberg group, used by Phong and Stein [29] to derive estimates for both the Bergman and Szegö projections, cannot yet be extended because the theory of singular integrals on weakly pseudoconvex surfaces is still in its early stages $[28,15]$. Similarly one does not know how to carry over the Fourier integral operator approach of Boutet de Monvel and Sjöstrand [10] to the weakly pseudoconvex case. Explicit construction of Cauchy-Fantappiè forms,

Received by the editors March 11, 1986. Paper presented April 11, 1986 to the 826th meeting of the American Mathematical Society, Indianapolis, special session on Several Complex Variables.

1980 Mathematics Subject Classification (1985 Revision). Primary 32A25, 32H10.

Partially supported by National Science Foundation grants MCS-8201063 and DMS-8501758. 
à la Kerzman and Stein [21], seems intractable in view of the complicated boundary geometry of weakly pseudoconvex domains.

In principle estimates for the Szegö projection can be derived from estimates for the $\bar{\partial}_{b}$ operator. Since the first version of this paper was written some details have become available [36, 37] of Kohn's microlocal techniques for proving subelliptic estimates for $\bar{\partial}_{b}$. These estimates provide an alternate approach to the local estimates at points of finite type given in $\$ 5$. (However the exact relation between Kohn's definition of finite type and D'Angelo's definition-the one I use-is presently unknown.) As far as I am aware, the global estimates for the Szegö projection in $\$ 4$ cannot be obtained directly from known estimates for $\bar{\partial}_{b}$.

This paper completely avoids the theory of the $\bar{\partial}_{b}$ operator by basing the proofs directly on the $\bar{\partial}$-Neumann problem. The idea is to transfer the Szegö projection to an interior operator (say by Green's theorem). This involves taking a derivative, so what has to be shown is that integrating against the Szegö kernel over the interior is smoothing by one derivative. This turns out to be equivalent to showing that integrating against the Bergman kernel over the boundary loses exactly one derivative. The latter property is demonstrated by relating the Bergman kernel to the Neumann operator in the usual way.

This method of relating a kernel function to the Bergman kernel is one I have used previously [9] to study Sobolev space analogues of the Bergman projection. This article is the promised companion paper, advertised in [9] under the title "Regularity of the Szegö projection in domains of finite type." In particular Appendix B contains proofs of some lemmas that were stated without proof in [9].

The main results of this paper are in $\$ \S 4$ and 5. $\S 2$ is devoted to a general lemma on stability of the Szegö projection. $\$ 1$ sets notation, and $\S 3$ recapitulates the $\bar{\partial}$-Neumann theory. The appendices are technical: Appendix A states a useful trick for localizing estimates, and Appendix B discusses Sobolev norms of harmonic functions.

1. Preliminaries. Throughout the paper $\Omega$ denotes a smooth bounded domain in $\mathbf{R}^{N}$, so

$$
\Omega=\left\{x \in \mathbf{R}^{N}: \rho(x)<0\right\}
$$

where $\rho: \mathbf{R}^{N} \rightarrow \mathbf{R}$ is a bounded $C^{\infty}$ function with the gradient $\nabla \rho$ nonvanishing on the boundary $b \Omega$. Generally $N$ equals $2 n$ and $\mathbf{R}^{N}$ is identified with $\mathbf{C}^{n}$. It is implicitly assumed that $n$ is bigger than one, since in $\mathbf{C}$ the results follow from classical potential theory. The usual Hilbert spaces of square-integrable functions $L^{2}(\Omega)$ and $L^{2}(b \Omega)$ carry the standard inner product and norm:

$$
\begin{aligned}
& \langle f, g\rangle=\int_{\Omega} f \bar{g} \text { and }\|f\|_{0}^{2}=\langle f, f\rangle \text { for } f \text { and } g \text { in } L^{2}(\Omega) ; \\
& \langle f, g\rangle_{h}=\int_{h \Omega} f \bar{g} \text { and }\|f\|_{0}^{2}=\langle f, f\rangle_{h} \text { for } f \text { and } g \text { in } L^{2}(b \Omega) .
\end{aligned}
$$

The same inner product notation is used to denote the action of a distribution $f$ on a smooth function $\bar{g}$. 
More generally the two-bar norm \|\|$_{s}$ denotes the norm in the interior Sobolev space $W^{s}(\Omega)$ and the three-bar norm denotes the norm in the boundary Sobolev space $W^{s}(b \Omega)$. (Here $W^{s}$ is the standard Sobolev space $[1,26]$ which, when $s$ is a positive integer, consists of functions with $s$ square-integrable derivatives.) The union over all real $s$ of $W^{s}(\Omega)$, with the usual inductive limit topology, is the space $W^{-\infty}(\Omega)$; the intersection, with the projective limit topology, is the space $C^{\infty}(\bar{\Omega})$ of functions smooth up to the boundary.

When $s \geqslant 0$,

$$
\|f\|_{-s}=\sup \left\{|\langle f, g\rangle|: g \in C_{0}^{\infty}(\Omega),\|g\|_{s}=1\right\} .
$$

Often one wants to pair not with compactly supported functions but with functions in $C^{\infty}(\bar{\Omega})$. I use the nonstandard notation

$$
\|f\|_{-s}^{*}=\sup \left\{|\langle f, g\rangle|: g \in C^{\infty}(\bar{\Omega}),\|g\|_{s}=1\right\}, \quad\|f\|_{s}^{*}=\|f\|_{s}
$$

when $s \geqslant 0$. The asterisk is a signal that close attention is required. It is an observation of Bell (for holomorphic [2] and pluriharmonic [5] functions) and of Straube (for the general case $[33,34]$ ) that \|\|$_{-s}^{*}$ is equivalent to \|\|$_{-s}$ on harmonic functions. In Appendix B a local equivalence is demonstrated.

I use the prefixes $\mathrm{Har}$ and $\mathrm{Hol}$ to indicate the subspaces of a function space consisting of harmonic and holomorphic functions respectively. Thus the Bergman projection $\mathbf{B}$ is the orthogonal projection from $L^{2}(\Omega)$ onto the closed subspace $\mathrm{Hol} L^{2}(\Omega)$; it is represented by integration against the Bergman kernel function $B(w, z)$ :

$$
\mathbf{B} f(w)=\langle f(\cdot), B(\cdot, w)\rangle .
$$

The Szegö projection $\mathbf{S}$ is the orthogonal projection from $L^{2}(b \Omega)$ onto the closed subspace $\mathrm{Hol} L^{2}(b \Omega)$ consisting of functions in $L^{2}(b \Omega)$ whose Poisson integrals are holomorphic in $\Omega$. It is represented by integration against the Szegö kernel function $S(w, z)$ :

$$
\mathbf{S} f(w)=\langle f(\cdot), S(\cdot, w)\rangle_{b} .
$$

Since for every real $s$ the spaces $\operatorname{Har} W^{s}(\Omega)$ and $W^{s-1 / 2}(b \Omega)$ are identified via the Poisson integral ([26]; also see Appendix B of this paper), the Szegö projection may be viewed as a map $\operatorname{Har} W^{1 / 2}(\Omega) \rightarrow \operatorname{Hol} W^{1 / 2}(\Omega)$ of interior spaces.

Indeed the approach in this paper is to study the Szegö projection by working on the interior of $\Omega$. It is useful to keep in mind the following version of Green's theorem. Fix a defining function $\rho$ and a smooth function $\psi$ equal to $|\nabla \rho|^{-1}$ in a neighborhood of $b \Omega$. For small nonnegative $\varepsilon$ write

$$
\Omega_{\varepsilon}=\{z \in \Omega: \rho(z)<-\varepsilon\} .
$$

If $u$ and $f$ are functions smooth up to the boundary of $\Omega_{\varepsilon}$ and $u$ is harmonic, then

$$
\int_{b \Omega_{\varepsilon}} \bar{u} f=\int_{\Omega_{\varepsilon}} \bar{u} \mathbf{L}_{\varepsilon} f
$$


where

$$
\mathbf{L}_{\varepsilon} f=\Delta[f \psi(\rho+\varepsilon)]
$$

If $u$ is replaced by a holomorphic function $h$, then a further integration by parts gives

$$
\int_{b \Omega_{\varepsilon}} \bar{h} f=\int_{\Omega_{\varepsilon}} \bar{h} \mathbf{L}_{\varepsilon}^{\prime} f
$$

where

$$
\mathbf{L}_{\varepsilon}^{\prime} f=f \Delta[\psi(\rho+\varepsilon)]+4 \sum \frac{\partial f}{\partial z_{j}} \frac{\partial}{\partial \bar{z}_{j}}[\psi(\rho+\varepsilon)] .
$$

The following definition is formulated for use in the discussion of local estimates.

DEFINITION 1.1. A pair of standard cut-off functions means a pair of functions $\zeta_{1}$ and $\zeta_{2}$ in $C_{0}^{\infty}\left(\mathbf{R}^{N}\right)$ such that $\zeta_{2}$ is identically equal to 1 in a neighborhood of the support of $\zeta_{1}$. The distance $d$ between $\zeta_{1}$ and $\zeta_{2}$ is the lesser of 1 and the distance between the support of $\zeta_{1}$ and the support of $\left(1-\zeta_{2}\right)$.

The Titchmarsh convention on constants [35] is in effect: the letter $C$ denotes a constant that may change its identity at each new occurrence.

2. Stability of the Szegö projection. For the proof of Theorem 4.1 it is necessary to know that the Szegö projections for subdomains converge appropriately to the Szegö projection for $\Omega$. The analogous statement for the Bergman projection is known $[30,6,19]$. The proof for the Szegö kernel is slightly more involved, and for completeness I include it here.

THEOREM 2.1. Let $\Omega$ be a smooth bounded domain in $\mathbf{C}^{n}$ (not necessarily pseudoconvex) with defining function $\rho$. Let $\mathbf{S}_{\varepsilon}$ be the Szegö projection for the subdomain $\Omega_{\varepsilon}$, where

$$
\Omega_{\varepsilon}=\{z \in \Omega: \rho(z)<-\varepsilon\} .
$$

Suppose that for the positive real number $r$ the estimate

$$
\left\|\mathbf{S}_{\varepsilon} u\right\|_{W^{r}\left(b \Omega_{\varepsilon}\right)} \leqslant C\|u\| W^{r}\left(b \Omega_{f}\right)
$$

holds for all sufficiently small positive $\varepsilon$ and all $u$ in $W^{r}\left(b \Omega_{\varepsilon}\right)$, with $C$ independent of $\varepsilon$ and $u$. Then

$$
\|\mathbf{S} u\|_{W^{r}(b \Omega)} \leqslant C\|u\|_{W^{r}(b \Omega)}
$$

for all $u$ in $W^{r}(b \Omega)$.

Proof. The heart of the proof is the verification that the Szegö kernel function $S_{\varepsilon}(w, z)$ for the subdomain $\Omega_{\varepsilon}$ approximates $S(w, z)$ in the sense that

$$
\left\|S(w, \cdot)-S_{\varepsilon}(w, \cdot)\right\|_{L^{2}\left(b \Omega_{\varepsilon}\right)} \rightarrow 0
$$

as $\varepsilon \rightarrow 0$, the convergence being uniform for $w$ in any compact subset of $\Omega$. Note that since the Szegö kernel function is holomorphic in the first variable, it will follow automatically from $(2.1)$ that $\left\|D_{w}^{\alpha} S(w, \cdot)-D_{w}^{\alpha} S_{\varepsilon}(w, \cdot)\right\|_{L^{2}\left(b \Omega_{\varepsilon}\right)} \rightarrow 0$ as $\varepsilon \rightarrow 0$ for every multi-index $\alpha$. 
Suppose for the moment that (2.1) is known. Fix a positive number $\delta$, and let $u$ be in $\operatorname{Har} W^{r}(b \Omega)$. Then $\mathbf{S} u(w)-\mathbf{S}_{\varepsilon} u(w)$ converges to zero uniformly for $w$ in $\Omega_{\delta}$, and so do all its derivatives. Therefore

$$
\begin{aligned}
\|\mathbf{S} u\| W^{r+1 / 2}\left(\Omega_{\delta}\right) & =\lim _{\varepsilon \rightarrow 0}\left\|\mathbf{S}_{\varepsilon} u\right\|_{W^{r+1 / 2}\left(\Omega_{\delta}\right)} \leqslant \lim \sup \left\|\mathbf{S}_{\varepsilon} u\right\|_{W^{r+1 / 2}\left(\Omega_{\varepsilon}\right)} \\
& \leqslant C \lim \sup \|u\| W^{r+1 / 2}\left(\Omega_{\varepsilon}\right) \leqslant C\|u\| W^{r+1 / 2}(\Omega),
\end{aligned}
$$

with $C$ independent of $\delta$ and $u$. Now let $\delta$ tend to zero and use the identification of $W^{r}(b \Omega)$ and $\mathrm{Har} W^{r+1 / 2}(\Omega)$ to obtain the result. To prove the theorem it remains to check (2.1).

Verification of (2.1). Fix $w$ in $\Omega$ and let $f_{\varepsilon}$ denote the unique function in Hol $L^{2}\left(b \Omega_{\varepsilon}\right)$ minimizing the norm subject to the constraint that $f_{\varepsilon}(w)=1$. Then

$$
S_{\varepsilon}(w, z)=\overline{f_{\varepsilon}(z)} /\left\|f_{\varepsilon}\right\|_{L^{2}\left(b \Omega_{\varepsilon}\right)}^{2} .
$$

Part of what needs to be shown is that the $f_{\varepsilon}$ converge to the solution $f_{0}$ of the corresponding extremal problem for $\Omega$.

At any rate

$$
\int_{b \Omega_{\varepsilon}}\left|f_{\varepsilon}\right|^{2} \leqslant \int_{b \Omega_{\varepsilon}}\left|f_{0}\right|^{2}
$$

since $f_{0}$ is a candidate for the solution to the extremal problem for $\Omega_{\varepsilon}$. The right-hand side of (2.3) tends to the square of the $L^{2}(b \Omega)$ norm of $f_{0}$, so

$$
\int_{h \Omega_{\varepsilon}}\left|f_{\varepsilon}\right|^{2} \leqslant(1+\alpha(\varepsilon)) \int_{h \Omega}\left|f_{0}\right|^{2}
$$

where $\alpha(\varepsilon) \rightarrow 0$ as $\varepsilon \rightarrow 0$. In particular (2.4) implies that the $f_{\varepsilon}$ form a normal family in $\Omega$. Let $f_{\varepsilon(j)}$ be a subsequence converging uniformly on compact sets. The limit function $F$ is holomorphic and $F(w)=1$.

Let $f_{\varepsilon}^{*}$ denote the function in $\operatorname{Har} L^{2}(b \Omega)$ whose value on $b \Omega$ is obtained by pushing $f_{\varepsilon}$ out from $b \Omega_{\varepsilon}$ normally. Then

$$
\int_{b \Omega}\left|f_{\varepsilon}^{*}\right|^{2} \leqslant(1+\beta(\varepsilon)) \int_{b \Omega_{\varepsilon}}\left|f_{\varepsilon}\right|^{2}
$$

where $\beta(\varepsilon) \rightarrow 0$ as $\varepsilon \rightarrow 0$, and by (2.4) the $f_{\varepsilon}^{*}$ are uniformly bounded in $L^{2}(b \Omega)$. Let $j(k)$ be a subsequence such that the $f_{\varepsilon(j(k))}^{*}$ converge weakly in $L^{2}(b \Omega)$. Stability of the Poisson kernel and the uniform estimate (2.4) imply that $f_{\varepsilon}(z)-f_{\varepsilon}^{*}(z)$ tends to zero for each fixed $z$ in $\Omega$. Therefore the $f_{\varepsilon(j(k))}^{*}$ converge to $F$ in $\Omega$. Accordingly $F \in \mathrm{Hol} L^{2}(b \Omega)$ and, in view of (2.4) and (2.5)

$$
\begin{aligned}
\int_{b \Omega}|F|^{2} & \leqslant \liminf \int_{b \Omega_{\varepsilon(j(k))}}\left|f_{\varepsilon(j(k))}\right|^{2} \\
& \leqslant \lim \sup \int_{b \Omega_{\varepsilon}}\left|f_{\varepsilon}\right|^{2} \leqslant \int_{b \Omega}\left|f_{0}\right|^{2} .
\end{aligned}
$$


The uniqueness of the solution to the extremal problem together with (2.6) implies that $F=f_{0}$. Since $f_{0}$ is the unique limit point of the normal family $f_{\varepsilon}$, the $f_{\varepsilon}$ converge to $f_{0}$ uniformly on compact subsets of $\Omega$. Moreover by (2.6) $\left\|f_{\varepsilon}\right\|_{L^{2}\left(b \Omega_{\varepsilon}\right)} \rightarrow$ $\left\|f_{0}\right\|_{L^{2}(b \Omega)}$. It follows from the relation (2.2) between $S_{\varepsilon}$ and $f_{\varepsilon}$ that $S_{\varepsilon}(w, z) \rightarrow$ $S(w, z)$ uniformly in $z$ in compact sets for fixed $w$.

The reproducing property of $S_{\varepsilon}$ implies, for each fixed $w$ in $\Omega$,

$$
\left\|S(w, \cdot)-S_{\varepsilon}(w, \cdot)\right\|_{L^{2}\left(b \Omega_{\varepsilon}\right)}^{2}=S_{\varepsilon}(w, w)-2 S(w, w)+\int_{b \Omega_{\varepsilon}}|S(w, \cdot)|^{2} .
$$

Now $S_{\varepsilon}(w, w) \rightarrow S(w, w)$ by the pointwise convergence just demonstrated, and since $S(w, \cdot) \in \operatorname{Har} L^{2}(b \Omega)$ the third term on the right-hand side of (2.7) tends to $\int_{b \Omega}|S(w, \cdot)|^{2}$, which also equals $S(w, w)$. Hence (2.7) implies (2.1) for fixed $w$ in $\Omega$.

The uniformity in $w$ follows easily by reductio ad absurdum. Suppose there were a sequence of points $w_{1}, w_{2}, \ldots$ in $\Omega$ converging to a point $w_{\infty}$ in $\Omega$ and a sequence $\varepsilon(j)$ converging to zero such that

$$
\left\|S\left(w_{j}, \cdot\right)-S_{\varepsilon(j)}\left(w_{j}, \cdot\right)\right\|_{L^{2}\left(b \Omega_{\varepsilon(j)}\right)}>c>0
$$

for some $c$ independent of $j$. Since $\left\|\partial S(w, \cdot) / \partial w_{k}\right\|_{L^{2}(b \Omega)}$ is the norm of the functional $h \rightarrow \partial h(w) / \partial w_{k}$ on $\mathrm{Hol} L^{2}(b \Omega)$, it is bounded by a constant times an inverse power of the distance from $w$ to the boundary $b \Omega$. Hence by the mean-value theorem the norm of $S\left(w_{j}, \cdot\right)-S\left(w_{\infty}, \cdot\right)$ in $L^{2}(b \Omega)$ tends to zero as $j \rightarrow \infty$, and a fortiori

$$
\left\|S\left(w_{j}, \cdot\right)-S\left(w_{\infty}, \cdot\right)\right\|_{L^{2}\left(b \Omega_{f(j)}\right)} \rightarrow 0
$$

as $j \rightarrow \infty$. For the same reason

$$
\left\|S_{\varepsilon(j)}\left(w_{j}, \cdot\right)-S_{\varepsilon(j)}\left(w_{\infty}, \cdot\right)\right\|_{L^{2}\left(b \Omega_{\varepsilon(1)}\right)} \rightarrow 0
$$

as $j \rightarrow \infty$. The pointwise convergence already proved implies

$$
\left\|S\left(w_{\infty}, \cdot\right)-S_{\varepsilon(j)}\left(w_{\infty}, \cdot\right)\right\|_{L^{2}\left(h \Omega_{f(1)}\right)} \rightarrow 0
$$

as $j \rightarrow \infty$. Combining the last three statements (2.9), (2.10), and (2.11) gives a contradiction to the ansatz (2.8). This completes the proof of (2.1) and of the theorem.

3. Reminders on the $\bar{\partial}$-Neumann problem. In this section I summarize some known estimates for the $\bar{\partial}$-Neumann problem that are required to run the machine in the next two sections. The references are the seminal paper of Kohn and Nirenberg [24] and the important recent contributions of Catlin $[11,12,39]$. The reader is advised to skip to $\S \S 4$ and 5 and refer to these results as needed.

Consider $\bar{\partial}$ as a closed densely defined operator acting on forms with square-integrable coefficients. The Hilbert space adjoint is denoted $\bar{\partial} *$. When $\Omega$ is a smooth bounded pseudoconvex domain in $\mathbf{C}^{n}$ the complex Laplacian $\bar{\partial} \bar{\partial} *+\bar{\partial} * \bar{\partial}$ acting on the space of $(0,1)$-forms is surjective; its inverse is the Neumann operator $N$. The quadratic form $Q$ is defined on $(0,1)$-forms in the domains of $\bar{\partial}$ and $\bar{\partial}^{*}$ by

$$
Q(f, f)=\|\bar{\partial} f\|_{0}^{2}+\left\|\bar{\partial}^{*} f\right\|_{0}^{2}
$$


One says that a compactness estimate holds for the $\bar{\partial}$-Neumann problem if for every positive $\varepsilon$ there exists $C(\varepsilon)$ such that

$$
\|f\|_{0}^{2} \leqslant \varepsilon Q(f, f)+C(\varepsilon)\|f\|_{-1}^{2} .
$$

Theorem 3.1 (Kohn AND Nirenderg). Suppose that $\Omega$ is a smooth bounded pseudoconvex domain in $\mathbf{C}^{n}$ such that a compactness estimate holds for the $\bar{\partial}$-Neumann problem. Fix a positive real number $r$. Then for every positive $\varepsilon$ there is $C(\varepsilon)$ such that

$$
\|\bar{\partial} * N f\|_{r} \leqslant \varepsilon\|f\|_{r}+C(\varepsilon)\|f\|_{0}
$$

for all $(0,1)$-forms $f$ such that $\bar{\partial} f=0$.

In [12] Catlin found an important sufficient criterion for the existence of a compactness estimate. A domain $\Omega$ in $\mathbf{C}^{n}$ satisfies Catlin's property (P) if for every positive $M$ there exists a plurisubharmonic function $\lambda$ in $C^{\infty}(\bar{\Omega})$ such that $0 \leqslant \lambda \leqslant 1$ and there exists a positive $\delta$ so that

$$
\sum_{j, k=1}^{n} \frac{\partial^{2} \lambda(z)}{\partial z_{j} \partial \bar{z}_{k}} t_{j} \bar{t}_{k} \geqslant M|t|^{2}
$$

for all $t$ in $\mathbf{C}^{n}$ and all points $z$ in $\Omega$ at distance less than $\delta$ from the boundary.

Theorem 3.2 (CATLin). If $\Omega$ is a smooth bounded pseudoconvex domain in $\mathbf{C}^{n}$ satisfying property $(\mathrm{P})$ then a compactness estimate holds for the $\bar{\partial}-$ Neumann problem. Moreover if (3.2) holds for a certain $M$ then (3.1) holds for $\varepsilon$ equal to $40 / M$.

Inspection of the proof shows that $C(\varepsilon)$ depends only on $\varepsilon$, the diameter of $\Omega$, and the first derivatives of a cut-off function in $C_{0}^{\infty}(\Omega)$ that is identically equal to one at points at distance $\delta$ or greater from the boundary of $\Omega$.

In $\$ 4$ it will be necessary to know that Theorem 3.2 holds uniformly on approximating subdomains of $\Omega$. This fact is implicit in Catlin's work, but for the reader's convenience I include an explicit statement and proof. First recall that by a construction of Diederich and Fornæss [17] there is a smooth defining function $\rho$ for the pseudoconvex domain $\Omega$ such that $-(-\rho)^{\eta}$ is strictly plurisubharmonic inside $\Omega$ for all sufficiently small positive $\eta$. (Range later showed [31] that if $\theta(z)$ is any smooth defining function for $\Omega$ then $\theta(z) \exp \left(-K|z|^{2}\right)$ is a Diederich-Fornæss defining function for sufficiently large $K$.)

Proposition 3.3. Let $\Omega$ be a smooth bounded pseudoconvex domain in $\mathbf{C}^{n}$ with Diederich-Forncess defining function $\rho$. Suppose $\Omega$ satisfies Catlin's property $(\mathrm{P})$. Then for all sufficiently small positive $\beta$ the interior approximating domain $\Omega_{\beta}$ (consisting of all points $z$ in $\Omega$ for which $\rho(z)<-\beta$ ) admits a Kohn-Nirenberg compactness estimate

$$
\|f\|_{L^{2}\left(\Omega_{\beta}\right)}^{2} \leqslant \varepsilon Q_{\beta}(f, f)+C(\varepsilon)\|f\|_{W^{-1}\left(\Omega_{\beta}\right)}^{2}
$$

with $C(\varepsilon)$ independent of $\beta$.

Proof. What has to be shown, in view of Catlin's theorem, is that for every $M$ and every sufficiently small positive $\beta$ there exists a plurisubharmonic function $\lambda_{\beta}$ in $C^{\infty}\left(\bar{\Omega}_{\beta}\right)$ such that $0 \leqslant \lambda_{\beta} \leqslant 1$ and

$$
\sum_{j, k=1}^{n} \frac{\partial^{2} \lambda_{\beta}(z)}{\partial z_{j} \partial \bar{z}_{k}} t_{j} \bar{t}_{k} \geqslant M|t|^{2}
$$


for all $t$ in $\mathbf{C}^{n}$ and all $z$ for which $-\beta-\delta<\rho(z)<-\beta$, where $\delta$ is a positive number independent of $\beta$.

By hypothesis there is a positive $\delta_{0}$ and a plurisubharmonic function $\lambda_{0}$ in $C^{\infty}(\bar{\Omega})$ such that $0 \leqslant \lambda_{0} \leqslant 1$ and (3.2) holds when $-\delta_{0}<\rho(z)<0$. If $\beta \leqslant \delta_{0} / 2$ and $\lambda_{\beta}$ is taken to be $\lambda_{0}$ then the desired inequality (3.3) holds when $-\beta-\delta<\rho(z)<-\beta$ as long as $\delta$ is no greater than $\delta_{0} / 2$.

When $\beta>\delta_{0} / 2$ the required $\lambda_{\beta}$ can be constructed from the Diederich-Fornæss defining function $\rho$. Denote $-(-\rho)^{\eta}$ by $\mu$, where $\eta$ is a fixed number such that $0<\eta<1$ and such that $\mu$ is strictly plurisubharmonic in $\Omega$. Let $m$ denote the minimum eigenvalue of the complex Hessian of $\mu$ on the set of $z$ in $\Omega$ such that $\rho(z) \leqslant-\delta_{0} / 2$. Set $\lambda_{\beta}$ equal to $\exp \left[2 \mathrm{Mm}^{-1}\left(\mu+\beta^{\eta}\right)\right]$. Then $0 \leqslant \lambda_{\beta}(z) \leqslant 1$ when $z \in \Omega_{\beta}$, the function $\lambda_{\beta} \in C^{\infty}\left(\bar{\Omega}_{\beta}\right)$, and (3.3) holds when

$$
-\left(M^{-1} m \log \sqrt{2}\right)^{1 / \eta}-\beta<\rho(z)<-\beta .
$$

Combining the two cases shows the required $\lambda_{\beta}$ exists, with $\delta$ independent of $\beta$, if $\delta$ is the minimum of $\delta_{0} / 2$ and $\left(M^{-1} m \log \sqrt{2}\right)^{1 / \eta}$. This proves the proposition.

It is a well-known consequence of the Kohn-Nirenberg theory that, since the Bergman projection $\mathbf{B}=\mathrm{Id}-\bar{\partial}^{*} N \bar{\partial}$, a compactness estimate for the $\bar{\partial}-$ Neumann problem implies that the Bergman projection maps $W^{r}(\Omega)$ continuously into itself for each positive $r$. The proof of this fact was for some years a closely held secret among the cognoscenti, but a sketch has now appeared in print [23]. The constants involved in the estimates depend on the domain only through its diameter and a finite number of derivatives of the boundary defining function. Therefore the previous proposition implies the following estimate for $\mathbf{B}$, uniform over subdomains.

Proposition 3.4. Let $\Omega$ be a smooth bounded pseudoconvex domain in $\mathbf{C}^{n}$ with Diederich-Forncess defining function $\rho$, and suppose $\Omega$ satisfies Catlin's property (P). Then for every nonnegative real number $r$ there exists a constant $C$ such that for all sufficiently small positive $\varepsilon$ the Bergman projection $\mathbf{B}_{\varepsilon}$ for the subdomain $\Omega_{\varepsilon}$ (the set of $z$ for which $\rho(z)<-\varepsilon$ ) admits the estimate

$$
\left\|\mathbf{B}_{\varepsilon} f\right\|_{W^{r}\left(\Omega_{\varepsilon}\right)} \leqslant C\|f\|_{W^{r}\left(\Omega_{\epsilon}\right)} .
$$

A point $q$ in the boundary of $\Omega$ is said to have finite type in the sense of D'Angelo [14] if the supremum of the order of contact of complex varieties with the boundary of $\Omega$ at $q$ is finite. Catlin has shown $[13,39]$ that a point $q$ in the boundary of a smooth bounded pseudoconvex domain is of finite type if and only if the $\bar{\partial}$-Neumann problem is subelliptic near $q$. This means that there is a neighborhood $U$ of $q$ and a positive $\varepsilon$ such that

$$
\|\phi\|_{\varepsilon}^{2} \leqslant C\left(Q(\phi, \phi)+\|\phi\|_{0}^{2}\right)
$$

for all smooth $(0,1)$-forms $\phi$ in the domains of $\bar{\partial}$ and $\bar{\partial}^{*}$ and supported in $U$. According to the Kohn-Nirenberg theory such a subelliptic estimate implies a pseudo-local estimate for $\bar{\partial}^{*} N$. The result that will be used in $\S 5$ is the following. 
Proposition 3.5. Let $\Omega$ be a smooth bounded pseudoconvex domain in $\mathbf{C}^{n}$ for which the Neumann operator preserves the space of $(0,1)$-forms with coefficients in $C^{\infty}(\bar{\Omega})$. Suppose $q$ is a point of finite type in the boundary of $\Omega$. Then $\bar{\partial}^{*} N$ gains a fractional derivative near $q$ in the following sense: There is a neighborhood $U$ of $q$ and a positive number $\varepsilon$ such that for all nonnegative real numbers $r$ and $m$ there exists a constant $C$ such that for all standard cut-off functions $\eta_{1}$ and $\eta_{2}$ supported in $U$

$$
\left\|\eta_{1} \bar{\partial}^{*} N f\right\|_{r+\varepsilon} \leqslant C d^{-c}\left\|\eta_{1}\right\|_{c}\left(\left\|\eta_{2} f\right\|_{r}+\|f\|_{-m}^{*}\right)
$$

for all $\bar{\partial}$-closed $(0,1)$-forms $f$ with coefficients in $C^{\infty}(\bar{\Omega})$, where $d$ is the distance between $\eta_{1}$ and $\eta_{2}$ (Definition 1.1).

The global regularity hypothesis is included to ensure that the global term in (3.4) can be taken with arbitrary negative index, rather than with $L^{2}$ norm. This hypothesis is of course satisfied if Catlin's property $(\mathrm{P})$ holds. The dependence on $\eta_{1}$ and $\eta_{2}$ has the indicated form since in the proof derivatives of $\eta_{1}$ appear together with derivatives of a sequence of cut-off functions with supports intermediate between those of $\eta_{1}$ and $\eta_{2}$. The form of this dependence will be used in $\$ 5$ in connection with the iteration trick of Appendix A.

As before, estimates for the Neumann operator lead to estimates for the Bergman projection. The following statement will suffice for the application in $§ 5$.

Proposition 3.6. With the same hypotheses as in Proposition 3.5 the Bergman projection admits the estimate

$$
\left\|\eta_{1} \mathbf{B} f\right\|_{r} \leqslant C\left(\left\|\eta_{2} f\right\|_{r}+\|f\|_{-m}^{*}\right)
$$

for all $f$ in $C^{\infty}(\bar{\Omega})$. The constant $C$ depends on the nonnegative real numbers $r$ and $m$ and on the standard cut-off functions $\eta_{1}$ and $\eta_{2}$.

4. Global estimates for the Szegö projection. This section is devoted to the proof of the main result on global regularity of the Szegö projection.

THEOREM 4.1. Let $\Omega$ be a smooth bounded pseudoconvex domain in $\mathbf{C}^{n}$. Suppose $\Omega$ satisfies Catlin's property $(\mathrm{P})$, discussed in $\S 3$. Then the Szegö projection for $\Omega$ maps $C^{\infty}(b \Omega)$ continuously into itself, and more generally maps $W^{r}(b \Omega)$ continuously into itself for every positive real number $r$.

The theorem applies to the following types of domains:

(a) Strictly PSEUdoconvex Domains. For such domains regularity of the Szegö projection is well known $[29,21,10]$, and this known case will be used in the proof of the more general Theorem 4.1 .

(b) WEAKLY PSEUdOCONVEX DOMAINS WITH REAL ANALYTIC BOUNDARY. An example is the set of points $(z, w)$ in $\mathbf{C}^{2}$ such that $|z|^{2}+(\operatorname{Re} z)^{2}+|w|^{4}<1$. The theorem of course applies to the simpler example $|z|^{2}+|w|^{4}<1$, but since this domain has bicircular symmetry an easier proof of the regularity theorem is known for it $[8,32]$.

(c) Pseudoconvex DOMAINs of FInITE TYPE. These domains include those of categories (a) and (b). For domains of finite type a stronger local regularity theorem holds. This is the main result of the next section. 
(d) Catlin's "Weakly RegulaR" Domains. These include the domains of category (c). An example that is not of finite type is the set of points $(z, w)$ in $\mathbf{C}^{2}$ defined by the inequality

$$
\left(|z|^{2}+|w-1|^{2}\right) \exp \left[-\left(|z|^{2}+|w-1|^{2}\right)^{-1}\right]<1-|w|^{2} .
$$

This smooth bounded pseudoconvex domain is strictly pseudoconvex at every boundary point except the point $(0,1)$, where the Levi form degenerates to infinite order.

Proof OF Theorem 4.1. Let $\rho$ be a Diederich-Fornæss defining function for $\Omega$, so that for all sufficiently small positive $\varepsilon$ the interior domain $\Omega_{\varepsilon}$ such that $\Omega_{\varepsilon}=\{z \in \Omega$ : $\rho(z)<-\varepsilon\}$ has smooth strictly pseudoccnvex boundary. In view of Theorem 2.1 what needs to be shown is that when $r>0$

$$
\left\|\mathbf{S}_{\varepsilon} u\right\|_{W^{r}\left(b \Omega_{\varepsilon}\right)} \leqslant C\|u\| W^{r}\left(b \Omega_{\varepsilon}\right)
$$

for all $u$ in $W^{r}\left(b \Omega_{\varepsilon}\right)$. Of course since $\Omega_{\varepsilon}$ is strictly pseudoconvex it is known that an estimate of the form (4.1) holds for each $\varepsilon$ : the whole point here is that $C$ may be taken independent of $\varepsilon$.

Since the Bergman kernel function $B_{\varepsilon}(w, z)$ for $\Omega_{\varepsilon}$ is smooth up to the boundary $b \Omega_{\varepsilon}$ off the boundary diagonal [20], the equation

$$
T_{\varepsilon} f(w)=\int_{b \Omega_{\varepsilon}} B_{\varepsilon}(w, z) f(z)
$$

defines a map $T_{\varepsilon}$ from smooth functions on $b \Omega_{\varepsilon}$ to holomorphic functions on $\Omega_{\varepsilon}$. The following a priori estimates, uniform in $\varepsilon$, are central to the proof of the theorem.

Key global estimates. For every positive real number $r$ there is a constant $C$ such that for all sufficiently small positive $\varepsilon$

$$
\begin{aligned}
& \left\|T_{\varepsilon} f\right\|_{W^{r}\left(\Omega_{\varepsilon}\right)} \leqslant C\|f\|_{W^{r+1}\left(\Omega_{\varepsilon}\right)} \text { for } f \text { in } C^{\infty}\left(\bar{\Omega}_{\varepsilon}\right), \\
& \|h\|_{W^{r+1}\left(\Omega_{\varepsilon}\right)} \leqslant C\left(\left\|T_{\varepsilon} h\right\|_{W^{r}\left(\Omega_{\varepsilon}\right)}+\|h\|_{L^{2}\left(\Omega_{\varepsilon}\right)}\right) \text { for } h \text { in } \operatorname{Hol} C^{\infty}\left(\bar{\Omega}_{\varepsilon}\right) .
\end{aligned}
$$

It is straightforward, granted these estimates, to prove (4.1). Since when $r=0$ the inequality holds by definition with $C$ equal to 1 , it suffices by interpolation to prove (4.1) when $r$ is large, say when $r>1$. Suppose then $u \in \operatorname{Har} W^{r}\left(b \Omega_{\varepsilon}\right)$ and $r>1$. Let $u_{1}, u_{2}, \ldots$ be a sequence of harmonic functions in $C^{\infty}\left(\bar{\Omega}_{\varepsilon}\right)$ such that $u_{j} \rightarrow u$ in $W^{r}\left(b \Omega_{\varepsilon}\right)$ as $j \rightarrow \infty$. Since $\Omega_{\varepsilon}$ is strictly pseudoconvex, it is known a priori that $\mathbf{S}_{\varepsilon} u_{j} \in C^{\infty}\left(\bar{\Omega}_{\varepsilon}\right)$ and that $\mathbf{S}_{\varepsilon} u_{j} \rightarrow \mathbf{S}_{\varepsilon} u$ in $W^{r}\left(b \Omega_{\varepsilon}\right)$ as $j \rightarrow \infty$. Rewriting key estimate (4.3) in terms of boundary norms (cf. (B.2) of Appendix B) gives

$$
\left\|\mathbf{S}_{\varepsilon} u_{j}\right\|_{W^{r}\left(b \Omega_{\varepsilon}\right)} \leqslant C\left(\left\|T_{\varepsilon} \mathbf{S}_{\varepsilon} u_{j}\right\|_{W^{r-1}\left(b \Omega_{\varepsilon}\right)}+\left\|\mathbf{S}_{\varepsilon} u_{j}\right\|_{L^{2}\left(b \Omega_{\varepsilon}\right)}\right) \text {. }
$$

Since $T_{\varepsilon} \mathbf{S}_{\varepsilon} u_{j}=T_{\varepsilon} u_{j}$ by the definition of the Szegö projection, it follows from key estimate (4.2) that

$$
\left\|\mathbf{S}_{\varepsilon} u_{j}\right\|_{W^{r}\left(b \Omega_{\varepsilon}\right)} \leqslant C\left\|u_{j}\right\|_{W^{r}\left(b \Omega_{\varepsilon}\right)} .
$$

Now let $j \rightarrow \infty$ to obtain (4.1). 
Proof of THE Key global estimates. To obtain (4.2) write $T_{\varepsilon} f=\mathbf{B}_{\varepsilon} \mathbf{L}_{\varepsilon}^{\prime} f$, where $\mathbf{L}_{\varepsilon}^{\prime}$ is the special first order differential operator defined by (1.5). Then (4.2) is immediate from the estimate for the Bergman projection stated in Proposition 3.4.

To obtain (4.3) write $T_{\varepsilon} h=\mathbf{B L}_{\varepsilon} h=\mathbf{L}_{\varepsilon} h-\bar{\partial}^{*} N_{\varepsilon} \bar{\partial} \mathbf{L}_{\varepsilon} h$, where $\mathbf{L}_{\varepsilon}$ is the differential operator defined by (1.3). In view of Proposition 3.3 and Theorem 3.1 there is a compactness estimate for $\bar{\partial} * N_{\varepsilon}$ that is independent of $\varepsilon$, so

$$
\left\|\bar{\partial}^{*} N_{\varepsilon} \bar{\partial} \mathbf{L}_{\varepsilon} h\right\|_{W^{r}\left(\Omega_{\varepsilon}\right)} \leqslant \delta\left\|\bar{\partial} \mathbf{L}_{\varepsilon} h\right\|_{W^{r}\left(\Omega_{\varepsilon}\right)}+C(\delta)\left\|\bar{\partial} \mathbf{L}_{\varepsilon} h\right\|_{L^{2}\left(\Omega_{\varepsilon}\right)} .
$$

Since $\Delta h=0$, the operator $\mathbf{L}_{\varepsilon}$ looks first-order to $h$, and since $\bar{\partial} h=0$, the operator $\bar{\partial} \mathbf{L}_{\varepsilon}$ also looks first-order to $h$. Therefore

$$
\left\|\bar{\partial}^{*} N_{\varepsilon} \bar{\partial} \mathbf{L}_{\varepsilon} h\right\|_{W^{r}\left(\Omega_{\varepsilon}\right)} \leqslant K\left(\delta\|h\|_{W^{r+1}\left(\Omega_{\varepsilon}\right)}+C(\delta)\|h\|_{W^{1}\left(\Omega_{\varepsilon}\right)}\right) .
$$

Accordingly

$$
\left\|\mathbf{L}_{\varepsilon} h\right\|_{W^{r}\left(\Omega_{e}\right)} \leqslant\left\|T_{\varepsilon} h\right\|_{W^{r}\left(\Omega_{\varepsilon}\right)}+K\left(\delta\|h\|_{W^{r+1}\left(\Omega_{e}\right)}+C(\delta)\|h\|_{W^{1}\left(\Omega_{e}\right)}\right)
$$

with $K$ and $C(\delta)$ independent of $\varepsilon$. By part (a) of Lemma B.8 of Appendix B,

$$
\|h\|_{W^{r+1}\left(\Omega_{\varepsilon}\right)} \leqslant C\left(\left\|\mathbf{L}_{\varepsilon} h\right\|_{W^{r}\left(\Omega_{\varepsilon}\right)}+\|h\|_{L^{2}\left(\Omega_{\varepsilon}\right)}\right) .
$$

Combining this estimate with the previous one and taking $\delta$ sufficiently small gives

$$
\|h\|_{W^{r+1}\left(\Omega_{\varepsilon}\right)} \leqslant C\left(\left\|T_{\varepsilon} h\right\|_{W^{r}\left(\Omega_{\varepsilon}\right)}+\|h\| W^{1}\left(\Omega_{\varepsilon}\right)\right)
$$

with a new constant $C$ independent of $\varepsilon$. Since $r>0$,

$$
C\|h\|_{W^{1}\left(\Omega_{e}\right)} \leqslant \frac{1}{2}\|h\|_{W^{r+1}\left(\Omega_{e}\right)}+C^{\prime}\|h\|_{L^{2}\left(\Omega_{e}\right)}
$$

so (4.3) follows. This completes the proof of Theorem 4.1.

REMARK 4.2. The theorem has the following consequences:

(1) The Szegö kernel function for $\Omega$ has finite order growth at the boundary [8], and in particular the Szegö kernel function $S(w, z) \in C^{\infty}(\omega \times \bar{\Omega})$ for every relatively compact subset $\omega$ of $\Omega$. In domains of finite type a stronger property holds (Corollary 5.2).

(2) The Szegö projection extends (by duality) to a continuous map of the space of distributions on $b \Omega$ into itself, and the extension satisfies the estimate $\|\mathbf{S} u\| \|_{-r} \leqslant$ $C\|\| u \|_{-r}$. Moreover if $u \in \operatorname{Har} W^{-\infty}(b \Omega)$ then the distribution $\mathbf{S} u$ is the boundary value of the holomorphic function defined by

$$
\mathbf{S} u(w)=\lim _{\varepsilon \rightarrow 0} \int_{b \Omega_{\varepsilon}} S(w, z) u(z) .
$$

The last statement follows from work of Straube [33] on distribution boundary values of harmonic functions.

(3) The operators $T$ and $U$ defined by

$$
T h(w)=\int_{b \Omega} B(w, z) h(z), \quad U h(w)=\int_{\Omega} S(w, z) h(z)
$$

satisfy the identities

$$
\langle T h, g\rangle=\langle h, g\rangle_{b}, \quad\langle h, g\rangle=\langle U h, g\rangle_{b}
$$


for $h$ and $g$ in $\operatorname{Hol}^{\infty}(\bar{\Omega})$, as follows by approximating in the first case $g$ and in the second case $h$ by compactly supported functions and applying Fubini's theorem. Moreover these operators have the following properties.

COROLlary 4.3. Under the hypotheses of Theorem 4.1

(i) $T$ and $U$ are inverse isomorphisms of $\mathrm{Hol}^{\infty}(\bar{\Omega})$ onto itself;

(ii) $T$ and $U$ extend ( uniquely) to inverse isomorphisms of $\mathrm{Hol}^{-\infty}(\Omega)$ onto itself;

(iii) the extensions define mutually inverse isomorphisms

$$
T: \operatorname{Hol} W^{r+1}(\Omega) \rightarrow \operatorname{Hol} W^{r}(\Omega), \quad U: \operatorname{Hol} W^{r}(\Omega) \rightarrow \operatorname{Hol} W^{r+1}(\Omega)
$$

for every real number $r$.

Proof. The hypothesis implies that for every real number $r$ there is a constant $C$ such that for all $h$ in $\mathrm{Hol} W^{-\infty}(\Omega)$

$$
\begin{aligned}
& C^{-1}\|h\|_{r} \leqslant \sup \left\{|\langle h, g\rangle|: g \in \operatorname{Hol} C^{\infty}(\bar{\Omega}),\|g\|_{-r}=1\right\} \leqslant C\|h\|_{r}, \\
& C^{-1}\|h\|_{r} \leqslant \sup \left\{\left|\langle h, g\rangle_{b}\right|: g \in \operatorname{Hol} C^{\infty}(\bar{\Omega}),\|g\|_{-r}=1\right\} \leqslant C\|h\|_{r} .
\end{aligned}
$$

The first statement follows from the estimates for the Bergman projection and the density of $\mathrm{Hol} C^{\infty}(\bar{\Omega})$ in $\mathrm{Hol} W^{-\infty}(\Omega)$. It was first proved by Bell [4] in the strictly pseudoconvex case and generalized in $[6,25]$. The second statement follows easily from the estimates of Theorem 4.1 for the Szegö projection and the same density.

When $h \in \mathrm{Hol}^{\infty}(\bar{\Omega})$ identities (4.4) and estimates (4.5) and (4.6) together with the correspondence (B.2) between boundary and interior norms give

$$
C^{-1}\|h\|_{r+1} \leqslant\|T h\|_{r} \leqslant C\|h\|_{r+1}, \quad C^{-1}\|h\|_{r} \leqslant\|U h\|_{r+1} \leqslant C\|h\|_{r} .
$$

In particular $T$ and $U$ map Hol $C^{\infty}(\bar{\Omega})$ continuously into itself and, since $\operatorname{Hol} C^{\infty}(\bar{\Omega})$ is dense in $\operatorname{Hol} W^{-\infty}(\Omega)$, they extend uniquely to continuous maps of $\operatorname{Hol} W^{-\infty}(\Omega)$ into itself. It is clear from (4.4) that $T U=U T=\operatorname{Id}$ on $\operatorname{Hol} C^{\infty}(\bar{\Omega})$, and this relation persists for the extension to $\mathrm{Hol} W^{-\infty}(\Omega)$.

5. Local estimates at points of finite type. In the previous section it was shown that the Szegö projection of a function that is smooth on the whole boundary is again smooth on the whole boundary. In this section it is shown that the Szegö projection of a function that is smooth near a point of finite type is again smooth near that point. The proof of this local result follows the same outline as the proof of the global result. The stronger property of subellipticity replaces compactness, which makes it possible to localize the argument. One simplification occurs: since the global regularity is known a priori, it is not necessary to work on approximating interior domains.

THEOREM 5.1. Let $\Omega$ be a smooth bounded pseudoconvex domain in $\mathbf{C}^{n}$ satisfying Catlin's property (P). Let $q$ be a point of finite type in the boundary of $\Omega$. Then the Szegö projection of every distribution that is smooth near $q$ is again smooth near $q$.

More precisely, there exists a neighborhood $U$ of $q$ such that for all cut-off functions $\zeta_{1}$ and $\zeta_{2}$ in $C_{0}^{\infty}(U)$ with $\zeta_{2}$ identically equal to 1 in a neighborhood of the support of $\zeta_{1}$, and for all real numbers $r$ and $m$, there exists a constant $C$ such that

$$
\left\|\zeta_{2} \mathbf{S} u\right\|_{r} \leqslant C\left(\left\|\zeta_{2} u\right\|_{r}+\|u\|_{-m}\right)
$$

for all distributions $u$ in $W^{-\infty}(b \Omega)$. 
COROLlary 5.2. In the above situation the Szegö kernel function

$$
S(w, z) \in C^{\infty}(U \cap \bar{\Omega} \times \bar{\Omega} \backslash\{(w, z) \in b \Omega \times b \Omega: w=z\}) .
$$

That is, the Szegö kernel function is smooth in both variables jointly when one variable approaches a boundary point of finite type and the other variable approaches any other boundary point.

Proof of Theorem 5.1. Choose the neighborhood $U$ by Catlin's theorem so that a subelliptic estimate of order $\varepsilon$ holds, as described in Proposition 3.5. As in $\S 4$ consider the operator $T$ acting on functions in $C^{\infty}(b \Omega)$ via

$$
T u(w)=\int_{b \Omega} B(w, z) u(z) .
$$

The heart of the proof lies in the following a priori estimates.

KEY LOCAL ESTIMATES. For all standard cut-off functions $\zeta_{1}$ and $\zeta_{2}$ supported in $U$ and for all nonnegative real numbers $r$ and $m$ there is a constant $C$ such that

$$
\begin{array}{ll}
\left\|\zeta_{1} T u\right\|_{r} \leqslant C\left(\left\|\zeta_{2} u\right\|_{r+1}+\|u\|_{-m}\right) & \text { for all } u \text { in } C^{\infty}(b \Omega), \\
\left\|\zeta_{1} h\right\|_{r+1} \leqslant C\left(\left\|\zeta_{2} T h\right\|_{r}+\|h\|_{-m}\right) & \text { for all } h \text { in } \operatorname{Hol} C^{\infty}(\bar{\Omega}) .
\end{array}
$$

Assume for the moment the validity of these estimates. Let $\eta_{1}$ and $\eta_{2}$ be another pair of standard cut-off functions, with $\eta_{1}$ equal to 1 in a neighborhood of the support of $\zeta_{1}$ and $\zeta_{2}$ equal to 1 in a neighborhood of the support of $\eta_{2}$. If $u \in C^{\infty}(b \Omega)$ and $r \geqslant 1$ then (5.3) implies

$$
\left\|\zeta_{1} \mathbf{S} u\right\|_{r} \leqslant C\left(\left\|\eta_{1} T \mathbf{S} u\right\|_{r-1}+\|\mathbf{S} u\|_{-m}\right) .
$$

By the global regularity proved in $\S 4$,

$$
\|\mathbf{S} u\|_{-m} \leqslant C \mid\|u\|_{-m} .
$$

Moreover $T \mathbf{S}=T$ on smooth functions, so by (5.2)

$$
\left\|\eta_{1} T \mathbf{S} u\right\| \|_{r-1} \leqslant C\left(\left\|\eta_{2} u\right\|_{r}+\|u\| \|_{-m}\right) .
$$

Thus (5.1) holds for smooth functions $u$ when $r \geqslant 1$. To prove (5.1) for general $u$ it suffices to take a sequence $u_{1}, u_{2}, \ldots$ of smooth functions such that $u_{j} \rightarrow u$ in $W^{-m}(b \Omega)$ and $\eta_{2} u_{j} \rightarrow \eta_{2} u$ in $W^{r}(b \Omega)$ and pass to the limit.

To extend the result to arbitrary $r$ observe that, by the estimate just proved,

$$
\left\|\zeta_{1} \mathbf{S}\left[\left(1-\zeta_{2}\right) u\right]\right\|_{1} \leqslant C\|u\|_{-m} .
$$

Combined with global regularity this inequality yields, when $r<1$,

$$
\begin{aligned}
\left\|\zeta_{1} \mathbf{S} u\right\|_{r} & \leqslant C\left(\left\|\mathbf{S}\left(\zeta_{2} u\right)\right\|_{r}+\left\|\zeta_{1} \mathbf{S}\left[\left(1-\zeta_{2}\right) u\right]\right\|_{1}\right) \\
& \leqslant C\left(\left\|\zeta_{2} u\right\|_{r}+\|u\|_{-m}\right) .
\end{aligned}
$$

Proof of THE KeY lOCAL eStimates. It is convenient to shift from boundary to interior norms using Lemma B.5 of Appendix B. Thus

$$
\left\|\zeta_{1} T u\right\|_{r} \leqslant C\left(\left\|\eta_{1} T u\right\|_{r+1 / 2}+\|T u\|_{-m-1 / 2}\right) .
$$

If $u \in \operatorname{Har} C^{\infty}(\bar{\Omega})$ then $T u=T \mathbf{S} u$ and the global estimates for $T$ and $\mathbf{S}$ yield

$$
\|T u\|_{-m-1 / 2} \leqslant C\|\mathbf{S} u\|_{-m+1 / 2} \leqslant C\|u\|_{-m} .
$$


Therefore to prove (5.2) it is enough to estimate the first term on the right-hand side of (5.4). Write $T u=\mathbf{B} \mathbf{L}^{\prime} u$ where $\mathbf{B}$ is the Bergman projection and $\mathbf{L}^{\prime}$ is the special first-order differential operator of (1.5) with $\varepsilon$ set equal to zero. The estimate of Proposition 3.6 for $\mathbf{B}$ gives

$$
\left\|\eta_{1} T u\right\|_{r+1 / 2} \leqslant C\left(\left\|\eta_{2} \mathbf{L}^{\prime} u\right\|_{r+1 / 2}+\left\|\mathbf{L}^{\prime} u\right\|_{-m-1 / 2}^{*}\right) .
$$

By Lemmas B.6 and B.5

$$
\left\|\eta_{2} \mathbf{L}^{\prime} u\right\|_{r+1 / 2} \leqslant C\left(\left\|\zeta_{2} u\right\|_{r+1}+\|u\|_{-m}\right)
$$

and

$$
\left\|\mathbf{L}^{\prime} u\right\|_{-m-1 / 2}^{*} \leqslant C \mid\|u\|_{-m},
$$

so (5.2) follows.

In proving (5.3) I assume that the intermediate cut-off functions $\eta_{1}$ and $\eta_{2}$ have been constructed as in Appendix A, so that their derivatives can be estimated by inverse powers of the distance $d$ between $\zeta_{1}$ and $\zeta_{2}$. Write $T h=\mathbf{L} h-\bar{\partial} * N \bar{\partial} \mathbf{L} h$, where $\mathbf{L}$ is the operator of (1.3) with $\varepsilon$ set equal to zero. By Lemma B.8,

$$
\begin{aligned}
\left\|\zeta_{1} h\right\|_{r+1} & \leqslant C d^{-c}\left\|\zeta_{1}\right\|_{c}\left(\left\|\eta_{1} \mathbf{L} h\right\|_{r}+\|h\|_{-m}\right) \\
& \leqslant C d^{-c}\left\|\zeta_{1}\right\|_{c}\left(\left\|\eta_{1} T h\right\|_{r}+\left\|\eta_{1} \bar{\partial}^{*} N \bar{\partial} \mathbf{L} h\right\|_{r}+\|h\|_{-m}\right) .
\end{aligned}
$$

The subelliptic estimate (Proposition 3.5) now comes into play:

$$
\left\|\eta_{1} \bar{\partial}^{*} N \bar{\partial} \mathbf{L} h\right\|_{r} \leqslant C d^{-c}\left(\left\|\eta_{2} \bar{\partial} \mathbf{L} h\right\|_{r-\varepsilon}+\|\bar{\partial} \mathbf{L} h\|_{-m-1}^{*}\right)
$$

when $r \geqslant \varepsilon$. Combining the last two estimates and invoking Lemma B.6 gives

$$
\left\|\zeta_{1} h\right\|_{r+1} \leqslant C d^{-c}\left\|\zeta_{1}\right\|_{c}\left(\left\|\zeta_{2} T h\right\|_{r}+\left\|\zeta_{2} h\right\|_{r+1-\varepsilon}+\|h\|_{-m}\right) \text {. }
$$

By the iteration trick of Appendix A it is possible to absorb the middle term on the right-hand side. Passing from interior norms to boundary norms gives (5.3). This completes the proof of Theorem 5.1.

Proof of Corollary 5.2. Let $\zeta_{1}$ and $\zeta_{2}$ be cut-off functions as in Theorem 5.1, and let $Z$ be a closed ball disjoint from the support of $\zeta_{2}$. By Sobolev's lemma it is enough to show that for each multi-index $\alpha$ and each positive $r$

$$
\sup \left\{\left\|\zeta_{1} D_{z}^{\alpha} S(\cdot, z)\right\|_{r}: z \in Z \cap \Omega\right\}<\infty .
$$

As observed in [21], if $P$ denotes the Poisson kernel then $S(w, z)=\mathbf{S}[P(\cdot, z)](w)$. Therefore by Theorem 5.1 and the local correspondence of Lemma B.5 between boundary and interior norms

$$
\left\|\zeta_{1} D_{z}^{\alpha} S(\cdot, z)\right\|_{r} \leqslant C\left(\left\|\zeta_{2} D_{z}^{\alpha} P(\cdot, z)\right\|_{r-1 / 2}+\left\|D_{z}^{\alpha} P(\cdot, z)\right\|_{-m}\right) .
$$

The first term is finite when $z \in Z \cap \Omega$ since the Poisson kernel is smooth on $b \Omega \times \bar{\Omega}$ off the diagonal. The second term is bounded by

$$
\sup \left\{\left|D^{\alpha} u(z)\right|: z \in Z \cap \Omega, u \text { harmonic, }\|u\|_{m}=1\right\} \text {. }
$$

Since the boundary norm of a harmonic function dominates the interior norm, this is bounded independently of $z$ when $m>n+|\alpha|$. This completes the proof. 
REMARK. The seminal paper on kernel smoothness is [20]. The analogue of Corollary 5.2 for the Bergman kernel function is implicit there. Essentially Kerzman's argument shows that a kernel smoothness result holds as soon as a local estimate of the form (5.1) holds.

Appendix A: An iteration trick. A common situation that arises in the study of pseudo-local estimates is that one can prove for a linear operator $L$ an estimate of the form

$$
\left\|\zeta_{1} L f\right\|_{r} \leqslant C\left(\left\|\zeta_{2} f\right\|_{r}+\left\|\zeta_{2} L f\right\|_{r-\varepsilon}\right)
$$

where $\zeta_{2}$ is identically equal to 1 on the support of $\zeta_{1}$, and one would like to absorb the lower order error term to obtain

$$
\left\|\zeta_{1} L f\right\|_{r} \leqslant C\left(\left\|\zeta_{2} f\right\|_{r}+\|f\|_{-m}\right) .
$$

If (A.1) held for all $r$ then (A.2) would follow by an obvious iteration, lowering the index of the error term by $\varepsilon$ at each step. Typically, however, (A.1) is known only for positive $r$, so that the best obvious conclusion is (A.2) with $m=0$.

Nonetheless it is possible to derive (A.2) from (A.1) by a more sophisticated iteration scheme. One has to determine the dependence of the constant on the cut-off functions and study what happens as their supports squeeze together. This ingenious idea essentially goes back to Hopf [27] and Schauder [40]. I learned the trick from the paper [18] of Greene and Krantz, and the formulation I give here is patterned on theirs.

Let $\Omega$ be a smooth bounded domain in $\mathbf{R}^{N}$ and suppose $L$ is a continuous linear operator from $W^{-\infty}(\Omega)$ into itself. Fix real numbers $r$ and $m$, a norm $G$ (thought of as a global term) dominating \|\|$_{-m}$, a norm $M$ (thought of as the main term) such that $M(g f) \leqslant\|g\|_{c} M(f)$, an open set $U$, a positive number $\varepsilon$, and an operator $T$.

HyPOTHESIS. There is a constant $C$ such that for all standard cut-off functions $\zeta_{1}$ and $\zeta_{2}$ supported in $U$

$$
\left\|\zeta_{1} L f\right\|_{r} \leqslant C d^{-c}\left\|\zeta_{1}\right\|_{c}\left(M\left(\zeta_{2} T f\right)+G(f)+\left\|\zeta_{2} L f\right\|_{r-\varepsilon}\right)
$$

for all $f$ in a certain subspace of $W^{-\infty}(\Omega)$. Here $d$ is the distance between $\zeta_{1}$ and $\zeta_{2}$ (Definition 1.1). Note that $C$ is independent of the cut-off functions.

Conclusion. There is a (new) constant $C$ such that for all standard cut-off functions $\zeta_{1}$ and $\zeta_{2}$ supported in $U$

$$
\left\|\zeta_{1} L f\right\|_{r} \leqslant C d^{-c}\left\|\zeta_{1}\right\|_{c}\left(M\left(\zeta_{2} T f\right)+G(f)\right)
$$

for all functions $f$ in the given class for which $\left\|\zeta_{2} L f\right\|_{r}<\infty$. The constant $C$ is independent of the cut-off functions and of $f$.

Proof. Fix cut-off functions $\zeta_{1}$ and $\zeta_{2}$ for which it is desired to prove (A.4). Also fix a nonnegative smooth function $\phi$ supported in the unit ball and with integral equal to one. For each positive integer $j$ set

$$
\phi_{j}(x)=\left(2^{j+2} d^{-1}\right)^{N} \phi\left(2^{j+2} d^{-1} x\right) .
$$


Let $\eta_{j}$ denote the convolution of $\phi_{j}$ with the indicator function of the set of points in $U$ at distance at least $2^{-j} d$ from the support of $\left(1-\zeta_{2}\right)$. Then each $\eta_{j} \in C_{0}^{\infty}(U)$, the function $\eta_{1}$ is identically equal to one in a neighborhood of the support of $\zeta_{1}$, each $\eta_{j+1}$ is identically equal to one in a neighborhood of the support of $\eta_{j}$, and $\zeta_{2}$ is identically equal to one in a neighborhood of the support of each $\eta_{j}$.

The hypothesis (A.3) implies that

$$
\left\|\eta_{j} L f\right\|_{r} \leqslant C d^{-c} 2^{j c}\left(M\left(\zeta_{2} T f\right)+G(f)+\left\|\eta_{j+1} L f\right\|_{r-\varepsilon}\right)
$$

where $C$ now depends on derivatives of the mollifier $\phi$, but not on $j$ nor on $f$. Since $L$ is continuous on $W^{-\infty}(\Omega)$ there is $m^{\prime}$ such that

$$
\|L f\|_{-m^{\prime}} \leqslant C\|f\|_{-m} \text {. }
$$

By a standard fact about Sobolev norms [1] there is $C$ such that, when $0<\lambda<1$,

$$
\|g\|_{r-\varepsilon} \leqslant C\left(\lambda\|g\|_{r}+\lambda^{-c}\|g\|_{-m^{\prime}}\right) \text {. }
$$

Combining the last three inequalities gives

$$
\left\|\eta_{j} L f\right\|_{r} \leqslant C d^{-c} 2^{j c}\left(M\left(\zeta_{2} T f\right)+\lambda^{-c} G(f)+\lambda\left\|\eta_{j+1} L f\right\|_{r}\right)
$$

where $C$ depends on $L$ and $m$ through (A.6) and on $r$ and $\varepsilon$ through (A.7) but is independent of $\lambda, j$, and $f$.

Assuming without loss of generality that the $C$ in (A.8) is larger than $|r|$ plus the dimension $N$, define $\lambda$ in terms of this $C$ by

$$
\lambda=(2 C)^{-1} d^{c} 2^{-j c-c(c+1)}
$$

and write

$$
N(j)=2^{-j c(c+1)}\left\|\eta_{j} L f\right\|_{r}
$$

to obtain

$$
N(j) \leqslant \frac{1}{2} N(j+1)+\left[C d^{-c} 2^{c(c+1)}\right]^{c+1}\left[M\left(\zeta_{2} T f\right)+G(f)\right]
$$

with the same $C$ as in (A.8). Since $\zeta_{2} L f \in W^{r}(\Omega)$ by hypothesis, it follows that $N(j) \rightarrow 0$ when $j \rightarrow \infty$. Therefore (A.9) implies

$$
N(j) \leqslant 2\left[C d^{-c} 2^{c(c+1)}\right]^{c+1}\left[M\left(\zeta_{2} T f\right)+G(f)\right]
$$

for all $j$. In particular setting $j$ equal to 1 gives

$$
\left\|\eta_{1} L f\right\|_{r} \leqslant C d^{-c}\left(M\left(\zeta_{2} T f\right)+G(f)\right)
$$

with a new $C$. Since $\eta_{1}$ is identically equal to 1 on the support of $\zeta_{1}$, the conclusion (A.4) follows.

Appendix B: harmonic functions in $\mathbf{R}^{N}$. This appendix contains explicit statements and proofs of some facts about Sobolev norms of harmonic functions for which there does not seem to be a suitable reference, although some of the results are implicit in [26]. The applications in the body of this paper are only to holomorphic functions, but nonetheless it seems more natural to state the estimates for harmonic functions. The astute reader will realize that similar results hold for elliptic operators 
other than the Laplacian, but since this generalization leads far afield from the main goal I do not consider it here. In a previous paper [9] I stated and used without proof a number of the results of this appendix.

Three basic principles are central to the discussion. The first is that in questions of duality it suffices to pair harmonic functions with functions of compact support. This idea comes from work of Bell and of Straube, and I include some remarks on Bell's $\Phi^{s}$ and Straube's $T^{k}$ operators. The second principle is the well known fact that boundary and interior norms of harmonic functions are equivalent, with a shift of one-half degree of differentiability. The third principle is that to estimate the Sobolev norm of a harmonic function it suffices to consider derivatives in the direction normal to the boundary.

Throughout this appendix $\Omega$ denotes a smooth bounded domain in $\mathbf{R}^{N}$. The Sobolev spaces are defined as in $\S 1$. The basic estimate of elliptic theory says that if $r \geqslant 0$ then

$$
\|f\|_{r+2} \leqslant C\left(\|\Delta f\|_{r}+\|f\|_{r+3 / 2}\right)
$$

for all $f$ in $C^{\infty}(\bar{\Omega})$. It will be useful to have a version of this estimate for negative $r$. Lions and Magenes introduced a new norm in [26] to study elliptic theory in $W^{-s}$ spaces. A consequence of their theory is the key inequality

$$
C^{-1}\|u\|_{r} \leqslant\|u\|_{r-1 / 2} \leqslant C\|u\|_{r}, \quad u \text { harmonic, }
$$

for every real number $r$. However I prefer to extend (B.1) by duality, using the norm \|\|$^{*}$ defined in (1.1).

In the following lemma the first statement generalizes the elliptic estimate (B.1), the second statement is a converse, and the third compares the norm (1.1) to the usual one.

LEMMA B.1. For every real number $r$ there is a constant $C$ such that

$$
\begin{aligned}
& \|f\|_{r}^{*} \leqslant C\left(\|f\|_{r-1 / 2}+\|\Delta f\|_{r-2}^{*}\right) ; \\
& \|f\|_{r-1 / 2} \leqslant C\left(\|f\|_{r}+\|\Delta f\|_{r-2}^{*}\right) ; \\
& \|f\|_{r}^{*} \leqslant C\left(\|f\|_{r}+\|\Delta f\|_{r-2}^{*}\right)
\end{aligned}
$$

for all $f$ in $C^{\infty}(\bar{\Omega})$.

For harmonic functions estimate (B.5) reduces to the statement

$$
\|u\|_{-r}^{*} \leqslant C\|u\|_{-r} \text { when } r \geqslant 0 \text { and } u \text { is harmonic. }
$$

The existence of such an inequality was first discovered by Bell [2] for holomorphic functions. Its proof for harmonic functions results from the following ideas of Bell and Straube.

In [3] Bell observed that the harmonic Bergman projection $\mathbf{B}_{\text {har }}$ from $L^{2}(\Omega)$ onto the subspace of harmonic functions is bounded from $W^{r}(\Omega)$ into itself when $r \geqslant 0$. Moreover he constructed a linear differential operator $\Phi^{s}$ (called $L^{s}$ in [3]) of order 
$s(s+3) / 2$ with $C^{\infty}(\bar{\Omega})$ coefficients such that for all $f$ in $C^{\infty}(\bar{\Omega})$

(B.7) $\quad \mathbf{B}_{\text {har }} \Phi^{s} f=\mathbf{B}_{\text {har }} f$;

(B.8) $\Phi^{s} f$ vanishes to order $s$ at $b \Omega$, that is, $\Phi^{s} f \in W_{0}^{s}(\Omega)$;

(B.9) $\Phi^{s} f$ is a sum of terms of the form $b \rho^{k} D^{\alpha} f$ where $\rho$ is the defining function for the domain, $b \in C^{\infty}(\bar{\Omega})$, and $|\alpha|=k$.

Bell's construction can be modified in the following way to obtain such an operator of order only $2 s$. Let $\psi$ be a smooth function equal to $|\nabla \rho|^{-1}$ in a neighborhood of $b \Omega$. Let $\Phi^{0}$ denote the identity and define $\Phi^{s}$ recursively by the formula

$$
\Phi^{s+1}=\Phi^{s} f-(s+2)^{-1}(s+1)^{-1} \Delta\left[\left(\Phi^{s} f\right)(\psi \rho)^{2}\right] .
$$

Then property (B.9) is obvious. Property (B.7) holds since if $F$ is any smooth function then integration by parts implies that $\Delta\left(\rho^{2} F\right)$ is orthogonal to the harmonic functions. To verify (B.8) observe that, modulo tangential derivatives, the principal part of the Laplacian at the boundary equals the second derivative in the normal direction.

I claim that $\Phi^{s}$ extends to be a bounded operator from $\operatorname{Har} W^{r}(\Omega)$ into $W^{r}(\Omega)$ when $r \geqslant 0$. The corresponding observation for the analogous operator on holomorphic functions is due to Bell [2], but in [3] he did not check this property for the harmonic $\Phi^{s}$. It suffices by a density argument to prove the following a priori estimate.

LEMMA B.2. For every nonnegative real number $r$ and every positive integer $s$ there is a constant $C$ such that $\left\|\Phi^{s} u\right\|_{r} \leqslant C\|u\|_{r}$ for all harmonic functions $u$ in $C^{\infty}(\bar{\Omega})$.

By interpolation it suffices to prove the result for integral $r$. In view of property (B.9) of $\Phi^{s}$ it suffices to prove the following estimate.

LEMMA B.3. For every nonnegative integer $k$ there is a constant $C$ such that $\left\|\rho^{k} u\right\|_{k} \leqslant C\|u\|_{0}$ for all harmonic functions $u$ in $C^{\infty}(\bar{\Omega})$.

PROOF. When $k=0$ there is nothing to prove. When $k=1$ integration by parts and the harmonicity of $u$ give

$$
\begin{aligned}
\|\rho u\|_{1}^{2} & =\|\rho u\|_{0}^{2}+\sum_{j=1}^{N} \int_{\Omega} D_{j}(\rho u) D_{j}(\rho \bar{u}) \\
& =\|\rho u\|_{0}^{2}+\sum_{j=1}^{N} \int_{\Omega}\left(D_{j} \rho\right)^{2}|u|^{2} \leqslant C\|u\|_{0}^{2} .
\end{aligned}
$$

Suppose now that the result is known for all nonnegative integers less than $k$, where $k \geqslant 2$. Then

$$
\left\|\rho^{k} u\right\|_{k} \leqslant C\left\|\Delta\left(\rho^{k} u\right)\right\|_{k-2} \leqslant C\left(\left\|\rho^{k-1} u\right\|_{k-1}+\left\|\rho^{k-2} u\right\|_{k-2}\right) \leqslant C\|u\|_{0} .
$$

Therefore the lemma holds by induction on $k$. 
Both E. J. Straube and P. D. Smith have communicated to me alternative proofs and generalizations of Lemma B.3. Also E. Ligocka [38] has found proofs of Lemmas B.2, B.3, and B.4.

In $[33,34]$ Straube constructed for each nonnegative integer $k$ an operator $T^{k}$ such that $\mathbf{B}_{\text {har }} T^{k}=\mathbf{B}_{\text {har }}$ and such that $T^{k}$ maps $W^{r}(\Omega)$ continuously into itself for every nonnegative $r$ and maps $W^{r}(\Omega)$ continuously into $W_{0}^{r}(\Omega)$ when $0 \leqslant r \leqslant k$. In view of the previous discussion an alternative way to define $T^{k}$ is $T^{k}=\Phi^{k} \mathbf{B}_{\mathrm{har}}$. The point of these ideas of Bell and Straube is the proof of (B.6), which I now restate for emphasis.

LEMMA B.4. For every nonnegative real number $r$ there exists a constant $C$ such that

$$
\|u\|_{-r} \leqslant\|u\|_{-r}^{*} \leqslant C\|u\|_{-r}
$$

for all harmonic functions $u$ in $C^{\infty}(\bar{\Omega})$.

Proof. The left-hand side is immediate from the definitions. To prove the right-hand side observe that if $u \in \operatorname{Har} C^{\infty}(\bar{\Omega})$ and $g \in C^{\infty}(\bar{\Omega})$ then choosing $k$ to be an integer greater than $r$ gives

$$
|\langle u, g\rangle|=\left|\left\langle u, T^{k} g\right\rangle\right| \leqslant\|u\|_{-r}\left\|T^{k} g\right\|_{r} \leqslant C\|u\|_{-r}\|g\|_{r} .
$$

Taking the supremum over $g$ in the unit ball of $W^{r}(\Omega)$ gives the result.

With this special result for harmonic functions in hand it is easy to prove the more general Lemma B.1. It is useful to introduce the solution operator $Q$ for the Dirichlet problem for the Laplacian with zero boundary condition. That is, $Q f$ vanishes on $b \Omega$ and $\Delta Q f=f$. By the standard elliptic estimate (B.1),

$$
\|Q f\|_{r} \leqslant C\|f\|_{r-2} \text { if } r \geqslant 2 \text {. }
$$

On the other hand, if $r \geqslant 0$ and $f$ and $g$ are in $C^{\infty}(\bar{\Omega})$, then

$$
|\langle Q f, g\rangle|=|\langle f, Q g\rangle| \leqslant C\|f\|_{-r-2}^{*}\|Q g\|_{r+2} \leqslant C\|f\|_{-r-2}^{*}\|g\|_{r},
$$

whence

$$
\|Q f\|_{-r}^{*} \leqslant C\|f\|_{-r-2}^{*} \text { if } r \geqslant 0 .
$$

By interpolation [26, p. 76] it follows that

$$
\|Q f\|_{r}^{*} \leqslant C\|f\|_{r-2}^{*} \text { for all real } r \text {. }
$$

Proof of Lemma B.1. Write $f=(f-Q \Delta f)+Q \Delta f$. By (B.10),

$$
\|Q \Delta f\|_{r}^{*} \leqslant C\|\Delta f\|_{r-2}^{*},
$$

and since $f-Q \Delta f$ is harmonic Lemma B.4 and estimate (B.2) imply

$$
\|f-Q \Delta f\|_{r}^{*} \leqslant C\|f-Q \Delta f\|_{r-1 / 2}=C\|f\|_{r-1 / 2} .
$$

Thus (B.3) holds. Moreover (B.2) and (B.10) imply

$$
\begin{aligned}
\|f\|_{r-1 / 2} & =\|f-Q \Delta f\|_{r-1 / 2} \leqslant C\|f-Q \Delta f\|_{r} \\
& \leqslant C\left(\|f\|_{r}+\|\Delta f\|_{r-2}^{*}\right),
\end{aligned}
$$

so (B.4) holds. Finally (B.5) is a trivial consequence of (B.3) and (B.4). 
Using Lemma B.1 it is not hard to localize the estimates (B.2) and (B.6) stating that various norms of harmonic functions are equivalent.

LEMMA B.5. For all real numbers $r$ and $m$ there is a constant $C$ such that for all standard cut-off functions $\zeta_{1}$ and $\zeta_{2}$

$$
\begin{aligned}
& \left\|\zeta_{1} u\right\|_{r}^{*} \leqslant C d^{-c}\left\|\zeta_{1}\right\|_{c}\left(\left\|\zeta_{2} u\right\|_{r-1 / 2}+\|u\| \|_{-m}\right), \\
& \left\|\zeta_{1} u\right\|_{r-1 / 2} \leqslant C d^{-c}\left\|\zeta_{1}\right\|_{c}\left(\left\|\zeta_{2} u\right\|_{r}+\|u\|_{-m}\right), \\
& \left\|\zeta_{1} u\right\|_{r}^{*} \leqslant C d^{-c}\left\|\zeta_{1}\right\|\left(\left\|\zeta_{2} u\right\|_{r}+\|u\|_{-m}\right)
\end{aligned}
$$

for all harmonic functions $u$ in $C^{\infty}(\bar{\Omega})$, where $d$ is the distance between $\zeta_{1}$ and $\zeta_{2}$ (Definition 1.1).

Proof. Put $f$ equal to $\zeta_{1} u$ in Lemma B.1. Then it remains only to estimate the error term $\Delta\left(\zeta_{1} u\right)$. Since $\Delta u=0$ it follows by iterative use of (B.5) that

$$
\left\|\Delta\left(\zeta_{1} u\right)\right\|_{r-2}^{*} \leqslant C d^{-c}\left\|\zeta_{1}\right\|_{c}\left(\left\|\zeta_{2} u\right\|_{r}+\sum_{|\alpha| \leqslant q}\left\|\eta D^{\alpha} u\right\|_{r-2 q}^{*}\right)
$$

where $\eta$ is a cut-off function that is equal to 1 in a neighborhood of the support of $\zeta_{1}$. Taking $q$ so large that $r-q<-m$ and invoking the global estimate of Lemma B. 4 gives

$$
\left\|\Delta\left(\zeta_{1} u\right)\right\|_{r-2}^{*} \leqslant C d^{-c}\left\|\zeta_{1}\right\|_{c}\left(\left\|\zeta_{2} u\right\|_{r}+\|u\|_{-m}\right) .
$$

This proves (B.12) and (B.13).

In view of (B.13) it is enough to prove (B.11) without the asterisk. To do this use the iteration trick of Appendix A with $L$ and $T$ equal to the identity, $G$ equal to \|\|$_{-m}$, the norm $M$ equal to \|\|$_{r-1 / 2}$, the open set $U$ equal to $\mathbf{R}^{N}$, the number $\varepsilon$ equal to $\frac{1}{2}$, and the subspace of $W^{-\infty}(\Omega)$ equal to $\operatorname{Har} C^{\infty}(\bar{\Omega})$. By the elliptic estimate (B.3) and the estimate (B.13) already proved,

$$
\begin{aligned}
\left\|\zeta_{1} u\right\|_{r} & \leqslant C\left(\left\|\zeta_{1} u\right\|_{r-1 / 2}+\left\|\Delta\left(\zeta_{1} u\right)\right\|_{r-2}^{*}\right) \\
& \leqslant C d^{-c}\left\|\zeta_{1}\right\|_{c}\left(\left\|\mid \zeta_{2} u\right\|_{r-1 / 2}+\left\|\zeta_{2} u\right\|_{r-1 / 2}+\|u\|_{-m}\right) .
\end{aligned}
$$

Hence (B.11) follows by the iteration trick.

Next consider derivatives of harmonic functions. Each derivative $\left(\partial / \partial x_{j}\right)$ maps $W^{r}(\Omega)$ continuously into $W^{r-1}(\Omega)$ except when $r=\frac{1}{2}$. The poirt of the next lemma is that restricting attention to harmonic functions eliminates the exception.

LemmA B.6. Let $L$ be a linear differential operator of order $k$ with $C^{\infty}(\bar{\Omega})$ coefficients. For all real numbers $r$ and $m$ there is a constant $C$ such that for all standard cut-off functions $\zeta_{1}$ and $\zeta_{2}$

$$
\begin{aligned}
& \left\|\zeta_{1} L u\right\|_{r} \leqslant C d^{-c}\left\|\zeta_{1}\right\|_{c}\left(\left\|\zeta_{2} u\right\|_{r+k}+\|u\|_{-m}\right), \\
& \left\|\zeta_{1} L u\right\|_{r} \leqslant C d^{-c}\left\|\zeta_{1}\right\|_{c}\left(\left\|\zeta_{2} u\right\|_{r+k}+\|u\|_{-m}\right)
\end{aligned}
$$

for all harmonic functions $u$. 
Proof. By a density argument it is enough to consider $u$ in $\operatorname{Har} C^{\infty}(\bar{\Omega})$. By induction it suffices to consider $k$ equal to 1 . By the remark preceding the lemma only the case of $r$ equal to $-\frac{1}{2}$ in (B.14) is nontrivial; but since (B.14) and (B.15) are equivalent (by Lemma B.5), the result will be proved as soon as the case $r$ equal -1 of (B.15) is verified.

If $L$ is tangential at the boundary there is nothing to do, so the lemma reduces to showing that

$$
\left\|\zeta_{1} D_{\nu} u\right\|_{-1} \leqslant C d^{-c}\left\|\zeta_{1}\right\|_{c}\left(\left\|\zeta_{2} u\right\|_{0}+\|u\|_{-m}\right)
$$

where $D_{\nu}$ is the directional derivative in the direction normal to the boundary. If $v \in \operatorname{Har} C^{\infty}(\bar{\Omega})$ then

$$
\int_{b \Omega} \bar{v} \zeta_{1} D_{\nu} u=\int_{b \Omega} u D_{\nu}\left(\zeta_{1} \bar{v}\right)-\int_{\Omega} u \Delta\left(\zeta_{1} \bar{v}\right)
$$

by Green's theorem. Consider for a moment the special case of this identity with $\zeta_{1}$ identically equal to 1 on $\Omega$. It yields

$$
\left|\int_{b \Omega} \bar{v} D_{\nu} u\right| \leqslant\|u\|_{0}\left\|D_{\nu} v\right\|_{0} \leqslant C\|u\|_{0}\|v\|_{1},
$$

the second step following by the case $r$ equals 0 of (B.15), which is equivalent to the known $r$ equals $\frac{1}{2}$ case of (B.14). Taking the supremum over $v$ in the unit ball of $\operatorname{Har} W^{1}(b \Omega)$ gives the global case of (B.16). Now return to (B.17) to obtain

$$
\left|\int_{b \Omega} \bar{v} \zeta_{1} D_{\nu} u\right| \leqslant C d^{-c}\left\|\zeta_{1}\right\|_{c}\left(\left\|\zeta_{2} u\right\|_{0}+\|u\| \|_{-m}\right)\left(\|v\|_{1}+\left\|D_{\nu} v\right\|_{0}\right) .
$$

Invoking the global result just proved and taking the supremum over $v$ gives (B.16).

To compute the norm of a harmonic function it is enough to consider tangential derivatives, since the vanishing of the Laplacian makes it possible to convert normal derivatives to tangential ones. It is not quite so clear that the converse is true: it is enough to consider normal derivatives. That is the point of the next result.

LEMMA B.7. For all real numbers $r$ and $m$ and every positive integer $k$ there is $a$ constant $C$ such that for all standard cut-off functions $\zeta_{1}$ and $\zeta_{2}$

$$
\left\|\zeta_{1} u\right\|_{r} \leqslant C d^{-c}\left\|\zeta_{1}\right\|_{c}\left(\left\|\zeta_{2} D_{\rho}^{k} u\right\|_{r-k}+\|u\|_{-m}\right)
$$

for all harmonic functions $u$, where $D_{\rho}=\Sigma\left(\partial \rho / \partial x_{j}\right)\left(\partial / \partial x_{j}\right)$ and $d$ is the distance between $\zeta_{1}$ and $\zeta_{2}$.

Proof. Passing to the limit over interior approximating domains shows that there is no loss of generality in assuming that $u \in C^{\infty}(\bar{\Omega})$. It also suffices to show that

$$
\left\|\zeta_{1} L u\right\|_{r} \leqslant C d^{-c}\left\|\zeta_{1}\right\|_{c}\left(\left\|\zeta_{2} D_{\rho} L u\right\|_{r-1}+\left\|\zeta_{2} u\right\|_{r+k-2}+\|u\|_{-m}\right)
$$

for an arbitrary linear differential operator $L$ of order $k-1$ with $C^{\infty}(\bar{\Omega})$ coefficients: the result then follows by the iteration trick of Appendix A and induction on $k$. 
By the generalized elliptic estimate (B.3) and the previous two lemmas

$$
\left\|\zeta_{1} L u\right\|_{r} \leqslant C\left[\left\|\zeta_{1} L u\right\|_{r-1 / 2}+d^{-c}\left\|\zeta_{1}\right\|_{c}\left(\left\|\zeta_{2} u\right\|_{r+k-2}+\|u\|_{-m}\right)\right] .
$$

Now for $v$ in $\operatorname{Har} C^{\infty}(\bar{\Omega})$

$$
\begin{aligned}
\left|\int_{b \Omega}\right| \nabla \rho\left|\bar{v} \zeta_{1} L u\right|= & \left|\int_{\Omega} \bar{v}(\Delta \rho) \zeta_{1} L u+\bar{v} \rho \Delta\left(\zeta_{1} L u\right)+2 \bar{v} D_{\rho}\left(\zeta_{1} L u\right)\right| \\
\leqslant & C d^{-c}\left\|\zeta_{1}\right\|_{c}\left(\left\|\zeta_{2} u\right\|_{r+k-2}+\|u\|_{-m}\right)\left(\|v\|_{-r+1}+\|\rho v\|_{-r+2}^{*}\right) \\
& +C\left\|\zeta_{1} D_{\rho} L u\right\|_{r-1}^{*}\|v\|_{-r+1} .
\end{aligned}
$$

But by the general elliptic estimate

$$
\|\rho v\|_{-r+2}^{*} \leqslant C\|\Delta(\rho v)\|_{-r}^{*} \leqslant C\|v\|_{-r+1}
$$

and

$$
\begin{aligned}
\left\|\zeta_{1} D_{\rho} L u\right\|_{r-1}^{*} & \leqslant C\left(\left\|\zeta_{1} D_{\rho} L u\right\|_{r-1}+\left\|\Delta\left(\zeta_{1} D_{\rho} L u\right)\right\|_{r-3}^{*}\right) \\
& \leqslant C d^{-c}\left\|\zeta_{1}\right\|_{c}\left(\left\|\zeta_{2} D_{\rho} L u\right\|_{r-1}+\left\|\zeta_{2} u\right\|_{r+k-2}+\|u\|_{-m}\right),
\end{aligned}
$$

so taking the supremum over $v$ in the unit ball of $\operatorname{Har} W^{-r+1}(\Omega)$ gives

$$
\left\|\zeta_{1} L u\right\|_{r-1 / 2} \leqslant C d^{-c}\left\|\zeta_{1}\right\|_{c}\left(\left\|\zeta_{2} D_{\rho} L u\right\|_{r-1}+\left\|\zeta_{2} u\right\|_{r+k-2}+\|u\|_{-m}\right) .
$$

Inserting this inequality in (B.19) gives the required estimate (B.18).

It is an easy corollary that the special operator $\mathbf{L}_{\varepsilon}$ of (1.3) also determines the norm of a harmonic function. For simplicity of notation I omit the subscript on $\mathbf{L}$ when $\varepsilon=0$.

LEMMA B.8. For all real numbers $r$ and $m$ there is a constant $C$ such that

(a) for all sufficiently small positive $\varepsilon$,

$$
\|u\|_{W^{r}\left(\Omega_{\varepsilon}\right)} \leqslant C\left(\left\|\mathbf{L}_{\varepsilon} u\right\|_{W^{r-1}\left(\Omega_{\varepsilon}\right)}+\|u\| W^{-m}\left(\Omega_{\varepsilon}\right)\right)
$$

for all harmonic functions $u$ in $C^{\infty}\left(\bar{\Omega}_{\varepsilon}\right)$; and

(b) for all standard cut-off functions $\zeta_{1}$ and $\zeta_{2}$

$$
\left\|\zeta_{1} u\right\|_{r} \leqslant C d^{-c}\left\|\zeta_{1}\right\|_{c}\left(\left\|\zeta_{2} \mathbf{L} u\right\|_{r-1}+\|u\|_{-m}\right)
$$

for all harmonic functions $u$ in $C^{\infty}(\bar{\Omega})$.

Proof. To prove (b), let $\eta$ be a cut-off function intermediate between $\zeta_{1}$ and $\zeta_{2}$, constructed as in Appendix A. By the previous lemma

$$
\left\|\zeta_{1} u\right\|_{r} \leqslant C d^{-c}\left\|\zeta_{1}\right\|_{C}\left(\left\|\eta D_{\rho} u\right\|_{r-1}+\|u\|_{-m}\right) \text {. }
$$

By the definition (1.3) of $\mathbf{L}$,

$$
\left\|\eta D_{\rho} u\right\|_{r-1} \leqslant C\left(\|\eta \mathbf{L} u\|_{r-1}+\|u\|_{W^{r}(\omega)}+\|\eta \rho \nabla u \cdot \nabla \psi\|_{r-1}+\|\eta u\|_{r-1}\right),
$$

where $\omega$ is a relatively compact subdomain of $\Omega$ containing the set where $\psi$ vanishes. But $\|u\|_{W^{r}(\omega)} \leqslant C\|u\|_{-m}$ and by the general elliptic estimate (B.3) and Lemmas B.5 and B.6

$$
\|\eta \rho \nabla u \cdot \nabla \psi\|_{r-1} \leqslant C\|\Delta(\eta \rho \nabla u \cdot \nabla \psi)\|_{r-3}^{*} \leqslant C d^{-c}\left(\left\|\zeta_{2} u\right\|_{r-1}+\|u\|_{-m}\right) .
$$


Combining these inequalities gives

$$
\left\|\zeta_{1} u\right\|_{r} \leqslant C d^{-c}\left\|\zeta_{1}\right\|_{c}\left(\left\|\zeta_{2} \mathbf{L} u\right\|_{r-1}+\left\|\zeta_{2} u\right\|_{r-1}+\|u\|_{-m}\right)
$$

so the result follows by the iteration trick of Appendix A.

Part (a) is a consequence. Indeed since the elliptic estimates hold uniformly on $\Omega_{\varepsilon}$ for small $\varepsilon$ (see $\$ 8.1$ of Chapter 2 of [26]) it is clear that the previous lemmas hold uniformly on $\Omega_{\varepsilon}$. From the proof one sees that (b) also holds uniformly on interior approximating subdomains.

\section{REFERENCES}

1. R. A. Adams, Sobolev spaces, Academic Press, New York, 1975.

2. S. R. Bell, Biholomorphic mappings and the $\bar{\partial}-$ problem, Ann. of Math. (2) 114 (1981), 103-113.

3. __ A duality theorem for harmonic functions, Michigan Math. J. 29 (1982), 123-128.

4. __ A representation theorem in strictly pseudoconvex domains, Illinois J. Math. 26 (1982), 19-26.

5. A A Sobolev inequality for pluriharmonic functions, Proc. Amer. Math. Soc. 85 (1982), $350-352$.

6. Steven R. Bell and Harold P. Boas, Regularity of the Bergman projection and duality of holomorphic function spaces, Math. Ann. 267 (1984), 473-478.

7. Steve Bell and David Catlin, Boundary regularity of proper holomorphic mappings, Duke Math. J. 49 (1982), 385-396.

8. Harold P. Boas, Regularity of the Szegö projection in weakly pseudoconvex domains, Indiana Univ. Math. J. 34 (1985), 217-223.

9. $\quad$ Sobolev space projections in strictly pseudoconvex domains, Trans. Amer. Math. Soc. 288 (1985), 227-240.

10. L. Boutet de Monvel and J. Sjöstrand, Sur la singularité des noyaux de Bergman et de Szegö, Astérisque 34-35 (1976), 123-164.

11. David Catlin, Boundary invariants of pseudoconvex domains, Ann. of Math. (2) 120 (1984), 529-586.

12. __ Global regularity of the $\bar{\partial}$-Neumann problem, Proc. Sympos. Pure Math., vol. 41, Amer.

Math. Soc., Providence, R. I., 1984.

13. Necessary conditions for subellipticity of the $\bar{\partial}-$ Neumann problem, Ann. of Math. (2) 117 (1983), 147-171.

14. John P. D'Angelo, Real hypersurfaces, orders of contact, and applications, Ann. of Math. (2) 115 (1982), 615-637.

15. Katharine P. Diaz, The Szegö kernel as a singular integral kernel on a family of weakly pseudoconvex domains, Doctoral dissertation, Princeton Univ., 1986.

16. Klas Diederich and John Erik Fornæss, Boundary regularity of proper holomorphic mappings, Invent. Math. 67 (1982), 363-384.

17. Pseudoconvex domains: bounded strictly plurisubharmonic exhaustion functions, Invent. Math. 39 (1977), 129-141.

18. Robert E. Greene and Steven G. Krantz, Deformation of complex structures, estimates for the $\bar{\partial}$ equation, and stability of the Bergman kernel, Advances in Math. 43 (1982), 1-86.

19. Stability properties of the Bergman kernel and curvature properties of bounded domains, Recent Developments in Several Complex Variables, Princeton Univ. Press, Princeton, N. J., 1981.

20. Norberto Kerzman, The Bergman kernel function. Differentiability at the boundary, Math. Ann. 195 (1972), 149-158.

21. N. Kerzman and E. M. Stein, The Szegö kernel in terms of Cauchy-Fantappiè kernels, Duke Math. J. 45 (1978), 197-224.

22. J. J. Kohn, Boundary regularity of $\bar{\partial}$, Recent Developments in Several Complex Variables, Princeton Univ. Press, Princeton, N. J., 1981.

23. , A survey of the $\bar{\partial}-$ Neumann problem, Proc. Sympos. Pure Math., vol. 41, Amer. Math. Soc., Providence, R. I., 1984.

24. J. J. Kohn and L. Nirenberg, Non-coercive houndary value problems, Comm. Pure Appl. Math. 28 (1965), 443-492. 
25. Gen Komatsu, Boundedness of the Bergman projector and Bell's duality theorem, Tôhoku Math. J. 36 (1984), 453-467.

26. J. L. Lions and E. Magenes, Non-homogeneous boundary value problems and applications, vol. I. Springer-Verlag, Berlin and New York, 1972.

27. E. Hopf, Über den funktionalen, inshesondere den analytischen Charakter der Lösungen elliptischer Differentialgleichungen zweiter Ordnung, Math. Z. 34 (1931), 194-233.

28. A. Nagel, E. M. Stein and S. Wainger, Boundary hehavior of functions holomorphic in domains of finite type, Proc. Nat. Acad. Sci. U. S. A. 78 (1981), 6596-6599.

29. D. H. Phong and E. M. Stein, Estimates for the Bergman and Szegö projections on strongly. pseudo-convex domains, Duke Math. J. 44 (1977), 695-704.

30. I. Ramadanov, Sur une propriété de la fonction de Bergman, C. R. Acad. Bulgar. Sci. 20 (1967). $759-762$.

31. R. Michael Range, A remark on bounded strictly plurisubharmonic exhaustion functions, Proc. Amer. Math. Soc. 81 (1981), 220-222.

32. Emil J. Straube, Exact regularity of Bergman, Szegö and Sobolev space projections in non-pseudoconvex domains, Math. Z. 192 (1986), 117-128.

33. Harmonic and analytic functions admitting a distribution boundary value, Ann. Scuola Norm. Sup. Pisa 11 (1984), 559-591.

34. Emil J. Straube, Orthogonal projections onto subspaces of the harmonic Bergman space, Pacific J. Math. 123 (1986), 465-476.

35. E. C. Titchmarsh, The theory of functions, 2nd ed., Oxford Univ. Press, London, 1939.

36. J. J. Kohn, Estimates for $\bar{\partial}_{h}$ on pseudo-convex $C R$ manifolds, Pseudodifferential Operators and Applications, Proc. Sympos. Pure Math., vol. 43, Amer. Math. Soc., Providence, R. I., 1985, pp. 207-217.

37. J. J. Kohn, The range of the tangential Cauchy-Riemann operator, Duke Math. J. 53 (1986), 525-545.

38. E. Ligocka, The Sobolev spaces of harmonic functions, Studia Math. 84 (1986).

39. David Catlin, Subelliptic estimates for the $\bar{\partial}$-Neumann problem on pseudoconvex domains, Ann. of Math. (to appear).

40. J. Schauder, Über lineare elliptische Differentialgleichungen zweiter Ordnung, Math. Z. 38 (1934), $257-282$.

Department of Mathematics, Texas A \& M University, Colilege Station, Texas 77843 